\title{
Ordering in Triangular Lattice Ising Antiferromagnet Due to Dilution and Magnetic Field
}

\author{
M. ŽukoviČ* , M. BorovskÝ And A. BobÁK \\ Department of Theoretical Physics and Astrophysics, Faculty of Science, P.J. Šafárik University \\ Park Angelinum 9, 04154 Košice, Slovak Republic
}

\begin{abstract}
We study effects of an external magnetic field and random site dilution on the magnetic ordering in the geometrically frustrated triangular lattice Ising antiferromagnet by the use of an effective-field theory with correlations. In particular, we find that already a small amount of the quenched dilution locally relieves the frustration which in the presence of the external field is manifested by multiple splitting of a broad frustration-induced $1 / 3$ magnetization plateau. Depending on the field strength, the dilution can either decrease or increase the magnetization or even change its effect from decreasing to increasing.
\end{abstract}

PACS numbers: 05.50.+q, 64.60.De, 75.10.Hk, 75.30.Kz, 75.50.Ee, 75.50.Lk

\section{Introduction}

The geometrically frustrated Ising antiferromagnet on a two-dimensional triangular lattice has been exactly shown to display no ordering at any finite temperatures [1]. Random site dilution locally relieves frustration and the system can order into a spin-glass state [2]. In the presence of a uniform external magnetic field a pure system displays a line of a second-order transition from the phase with two sublattices aligned parallel and one antiparallel to the field at lower temperatures $(\uparrow \downarrow \uparrow)$ to the phase in which all spins are aligned parallel to the field at higher temperatures $(\uparrow \uparrow \uparrow)[3]$. This phase transition is at low temperatures manifested by a broad plateau with the height $1 / 3$ in magnetization vs. field curves, corresponding to the ferrimagnetic spin arrangement $(\uparrow \downarrow \uparrow)$. Such a plateau has also been observed experimentally in some frustrated antiferromagnetic compounds on triangular lattice, such as $\mathrm{Ca}_{3} \mathrm{Co}_{2} \mathrm{O}_{6}$, which below a certain temperature threshold splits into multistep dependence [4]. The origin of the splitting has been intensively studied but it remains controversial (see [5] and references therein). We expect that also the presence of nonmagnetic impurities, a small amount of which is a common feature in real magnetic materials, should significantly affect the plateau.

\section{Effective field theory}

Hence, the goal of the present work is to study effects of uniform site dilution on the magnetization processes in the spin-1/2 Ising antiferromagnet in a field on the 2D triangular lattice. The model Hamiltonian is given by

\footnotetext{
* corresponding author; e-mail: milan.zukovic@upjs.sk
}

$$
H=-J \sum_{\langle i, j\rangle} \xi_{i} \xi_{j} s_{i} s_{j}-h \sum_{i} \xi_{i} s_{i},
$$

where $s_{i}= \pm 1,\langle i, j\rangle$ is the sum over nearest neighbors (NNs), $h$ is the external magnetic field, $\xi_{i}$ is the random variable which takes the values of unity or zero depending on whether the site $i$ is occupied by magnetic atom or not and its configurational average is the concentration of magnetic sites $p$, and $J<0$ is the exchange interaction constant.

We employ an effective-field theory (EFT) with correlations in which all the single-site kinematic relations are correctly accounted for (see e.g., [6]). However, since in the EFT treatment all correlations between NNs of a selected central spin are completely neglected, straightforward application of EFT to the present system would lead to a complete loss of frustration and thus inevitably incorrect results. To avoid this, we split the lattice into three interpenetrating sublattices, as shown in Fig. 1. Then all NNs of a spin on one sublattice are from the other two sublattices and the frustration arises from the effort to simultaneously satisfy all the mutual(isotropic)

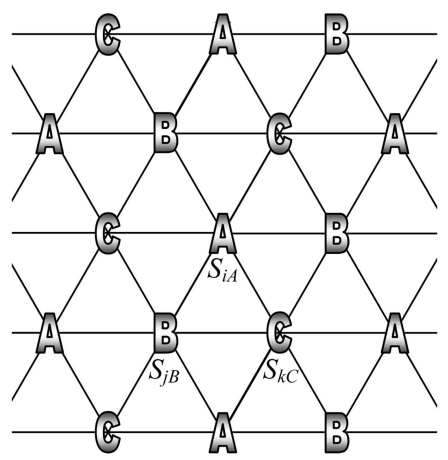

Fig. 1. Triangular lattice partition into three sublattices $A, B$ and $C$. 
intersublattice antiferromagnetic interactions. For example, if the spin $s_{i A}$ is in a state +1 then the energy is minimized if its NNs $s_{j B}=s_{k C}=-1$ (as well as the rest of NNs). However, for $s_{j B}=-1$ it would be energetically favorable if all its NNs, including $s_{i A}$ and $s_{k C}$, were in the state +1 , which results in frustration. Within the EFT framework [6], the respective sublattice magnetizations $m_{A}, m_{B}$ and $m_{C}$ can be calculated from the set of coupled equations

$$
\begin{aligned}
& m_{A}=\left.p\left(a+b m_{B}\right)^{3}\left(a+b m_{C}\right)^{3} \tanh (x+\beta h)\right|_{x=0}, \\
& m_{B}=\left.p\left(a+b m_{A}\right)^{3}\left(a+b m_{C}\right)^{3} \tanh (x+\beta h)\right|_{x=0}, \\
& m_{C}=\left.p\left(a+b m_{A}\right)^{3}\left(a+b m_{B}\right)^{3} \tanh (x+\beta h)\right|_{x=0},
\end{aligned}
$$

where $a=1-p+p \cosh (\beta J D), \quad b=\sinh (\beta J D)$, $\beta=1 / k_{\mathrm{B}} T$, and $D=\partial / \partial x$ is the differential operator. The total magnetization per site $m$ is defined as $m=\left(m_{A}+m_{B}+m_{C}\right) / 3$.

\section{Results and discussion}

We find that such a treatment indeed reproduces the correct behavior with no long-range order down to $T=0$ in zero field. In line with the previous Monte Carlo mean-field, Monte Carlo and renormalization-group results (see [3] and references therein), at low temperatures we observe the ferrimagnetic ordering $(\uparrow \downarrow \uparrow)$ with the broad $1 / 3$ magnetization plateau in the range $0<$ $h /|J|<6$ (Fig. 2). However, inclusion of already a very small amount of nonmagnetic impurities makes the broad plateau split into a number of smaller parts, forming an equidistant step-like dependence. This behavior is a sign of modulation of spin frustration by local destroying the ferrimagnetic spin arrangement. Thus, in the ferri-

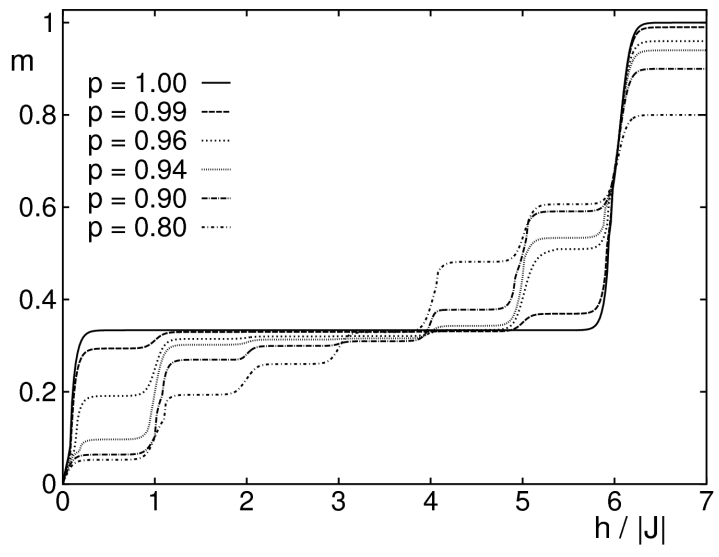

Fig. 2. Magnetization vs. magnetic field plots for different values of the concentration $p$ at $k_{\mathrm{B}} T /|J|=0.1$.

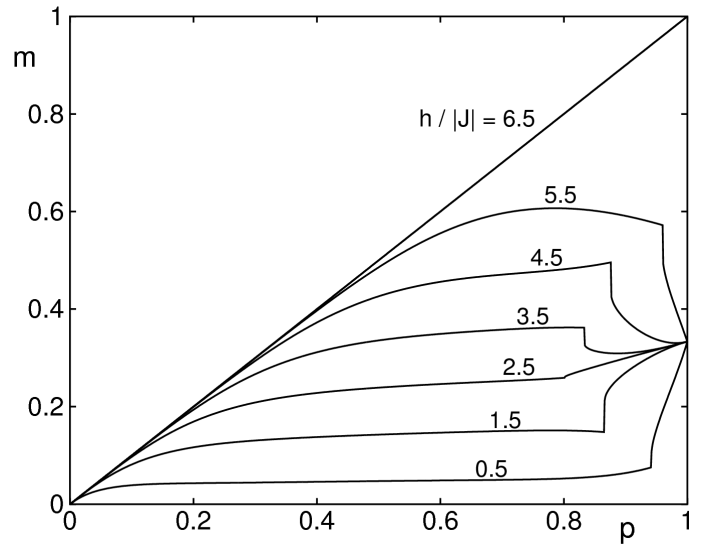

Fig. 3. Magnetization vs. concentration plots for different values of the field $h /|J|$ at $k_{\mathrm{B}} T /|J|=0.1$.

magnetic phase, increasing dilution can either decrease or increase the total magnetization, depending on the field value. The former tendency is observed at lower and the latter at higher fields. In Fig. 3 we can better see that at the intermediate fields (approximately within $3<h /|J|<5$ ) the magnetization can even reverse its trend: from the initial decrease it starts increasing upon further dilution.

We hope that this work would stimulate experimental studies to see if the above observed phenomena could be reproduced in the corresponding real compounds, such as $\mathrm{Ca}_{3} \mathrm{Co}_{2} \mathrm{O}_{6}$.

\section{Acknowledgments}

This work was supported by the Scientific Grant Agency of Ministry of Education of Slovak Republic (grant No. 1/0431/10).

\section{References}

[1] G.H. Wannier, Phys. Rev. 79, 357 (1950).

[2] G.S. Grest, E.F. Gabl, Phys. Rev. Lett. 43, 1182 (1979).

[3] R.R. Netz, A.N. Berker, Phys. Rev. Lett. 66, 377 (1991).

[4] V. Hardy, M.R. Lees, O.A. Petrenko, D.M. Paul, D. Flahaut, S. Hébert, A. Maignan, Phys. Rev. B 70, 064424 (2004).

[5] X. Yao, S. Dong, H. Yu, J. Liu, Phys. Rev. B 74, 134421 (2006).

[6] T. Kaneyoshi, Acta Phys. Pol. A 83, 703 (1993). 